\title{
Waveform and packet structure of lion roars
}

\author{
W. Baumjohann ${ }^{1}$, R. A. Treumann ${ }^{1}$, E. Georgescu ${ }^{1,2}$, G. Haerendel ${ }^{1}$, K.-H. Fornacon ${ }^{3}$, and U. Auster ${ }^{3}$ \\ ${ }^{1}$ Max-Planck-Institut für extraterrestrische Physik, Garching, Germany \\ ${ }^{2}$ Institut for Space Sciences, Bucharest, Romania \\ ${ }^{3}$ Institut für Geophysik und Meteorologie, TU Braunschweig, Germany
}

Received: 12 February 1999 / Revised: 12 May 1999 / Accepted: 18 May 1999

\begin{abstract}
The Equator-S magnetometer is very sensitive and has a sampling rate of normally $128 \mathrm{~Hz}$. The high sampling rate allows for the first time fluxgate magnetometer measurements of ELF waves between the ion cyclotron and the lower hybrid frequencies in the equatorial dayside magnetosheath. The so-called lion roars, typically seen by the Equator-S magnetometer at the bottom of the magnetic troughs of magnetosheath mirror waves, are near-monochromatic packets of electron whistler waves lasting for a few wave cycles only, typically $0.25 \mathrm{~s}$. They are right-hand circularly polarized waves with typical amplitudes of $0.5-1 \mathrm{nT}$ at around one tenth of the electron gyrofrequency. The cone angle between wave vector and ambient field is usually smaller than $1.5^{\circ}$.
\end{abstract}

Key words. Interplanetary physics (MHD waves and turbulence; plasma waves and turbulence)

\section{Introduction}

More than 20 years ago, Smith and Tsurutani (1976) published the first search-coil magnetometer observations of what they called lion roars inside magnetic troughs in the dayside magnetosheath. They found that the lion roars are narrow-banded right-hand polarized waves, basically electron cyclotron waves, that are relatively short-lived, about $2 \mathrm{~s}$, and that they have typical frequencies of about $120 \mathrm{~Hz}$ and typical amplitudes of $0.1 \mathrm{nT}$. Some years later, Tsurutani et al. (1982) studied the same phenomenon with the plasma wave instrument on ISEE. They found these waves at somewhat lower frequencies $(50-100 \mathrm{~Hz})$ and also established that lion roars are intimately related to

Correspondence to: W. Baumjohann, e-mail: bj@mpe.mpg.de mirror mode structures and that they are caused by the perpendicular electron pressure anisotropy in the magnetic troughs of these structures.

However, due to the instrumentation employed in the earlier studies, the actual waveform of the lion roars was unknown until very recently. Zhang et al. (1998) used the waveform capture instrument onboard Geotail to study $20-300 \mathrm{~Hz}$ lion roars with typical amplitudes of $0.1 \mathrm{nT}$ within mirror waves (their type A, similar to the lion roars analyzed in the earlier studies and the present paper) and similar waves without ambient magnetic field depletion closer to the bow shock (their type B). Zhang et al. (1998) did not discuss the wave form itself very much, but rather the angle between the wave vector, $\mathbf{k}$, and the ambient field. They did this by applying a minimum variance analysis to the magnetic waveform and removed the $\pm 180^{\circ}$-ambiguity of the $\mathbf{k}$-vector using electric field data. They obtained typical cone angles between the wave vector and the ambient field of $\theta_{k B} \approx 10^{\circ}$, which is lower than the typical values found by Smith and Tsurutani (1976), but still rather oblique for electron whistlers.

In the present paper we will also study the waveform of mirror trough lion roars, but using the fluxgate magnetometer onboard Equator-S. This will limit our study to waves below $64 \mathrm{~Hz}$, but extend the frequency range down to $8 \mathrm{~Hz}$. Using a different approach we will show for single cases as well as in statistics covering 356 cases that (1) lion roars can be found down to $8 \mathrm{~Hz}$ (the lower limit set in our analysis), (2) the lion roar wave forms have a clear packet structure, and (3) the average cone angle found in our frequency range, $\theta_{k b} \approx 0.3^{\circ}$, is much smaller than previously thought and that lion roars travel essentially parallel to the background field.

\section{Instrumentation and data}

The Equator-S magnetic field instrument is fully described in Fornacon et al. (1999). In short, it consists of two units with a pair of three-axes fluxgate magnetometers each. The sensors of the primary and the 
redundant units are mounted on two rigid booms, with the main sensor located at the end of the $1.8-\mathrm{m}$ boom and the other $50 \mathrm{~cm}$ further inboard. The sampling rate is 128 vectors/s in normal mode, when only the outboard magnetometer is used, and 64 vectors/s for dual mode operation. The amplitude resolution is 16 bit and ranges are selected automatically in steps of 4 between 256 and $64000 \mathrm{nT}$. The data used in the present study are mainly $128 \mathrm{~Hz}$ data, all sampled with a resolution of about $10 \mathrm{pT}$ in the $256 \mathrm{nT}$ mode.

For the four months the Equator-S encountered the near-magnetopause day- and morningside magnetosheath, we searched for all occurrences of mirror modes and looked into each of their troughs or minima to find lion roars. We were successful in 356 cases.

\section{Case studies}

Before proceeding to the full data set, we will first present six typical examples. The upper panel of Fig. 1 shows the magnetic amplitude of a magnetosheath mirror mode observed on 24 January 1998 . Note that the trough is rather deep, with $\Delta B / B \approx 1$, and that the field in the trough's center is slightly enhanced. Actually, we found that most mirror troughs had $\Delta B / B \approx 1$ and many had a stronger core field.

The lion roars can already be recognized in the unfiltered data (see Fig. 7 of Fornacon et al., 1999) but here we use high-pass filtered $(f \geq 4 \mathrm{~Hz})$ components in mean field-aligned coordinates (middle panels of Fig. 1). Three lion roar events, stretching over about $1 \mathrm{~s}$ each could be identified in the trough. The lion roars do not appear right at the center of the trough, but rather at its edges, on both sides of the stronger core field. In all three cases, the two transverse components are nearly identical but phase-shifted by $90^{\circ}$, indicating right-hand circular polarization of the wave, just as expected for electron whistlers.

Since the lion roar signals are rather short-lived, we use a wavelet analysis to determine spectrograms of the signals. The wavelet used is the so-called Morlet wavelet, which is best suited for this type of analysis (Lui and Najmi, 1997; Torrence and Compo, 1998). By transforming the complex signal $\left(B_{r, l}=B_{x} \pm i B_{y}\right)$, we calculate the right-hand and left-hand polarization spectrograms. The lower panel of Fig. 1 shows the spectrograms of the right-hand polarized signals.

The left-most diagrams show one of the strongest and longest-lived lion roar signal wave form we found in our data set, about 10 wave cycles with an amplitude of $\pm 1 \mathrm{nT}$. The signal is nearly monochromatic with a center frequency near $15 \mathrm{~Hz}$. This is less than $1 / 20$ of the electron gyrofrequency near $350 \mathrm{~Hz}$. The lion roar in the middle panels has a somewhat higher frequency of $21.5 \mathrm{~Hz}$ despite of the same background field magnitude. Moreover, it is clearly broken into 3-4 wave packets, some of them lasting only for 2-3 wave cycles. The third lion roar (in the right-most diagrams) has again a somewhat different center frequency $(18 \mathrm{~Hz})$.

We have also determined the wave normal direction as the direction of the minimum variance, i.e., along the eigenvector having the smallest eigenvalue in a minimum variance or principal component analysis (see, for example, Smith and Tsurutani, 1976; Zhang et al., 1998). The wave normal direction, i.e., its k-vector, was clearly aligned with the background magnetic field. The cone angle, $\theta_{k b}$, had values of $1.1^{\circ}, 0.1^{\circ}$, and $0.7^{\circ}$ during the three events.

A clear packet structure can also be seen in Fig. 2, which shows wave forms and wavelet spectrograms for two other lion roar wave packet trains inside a magnetic
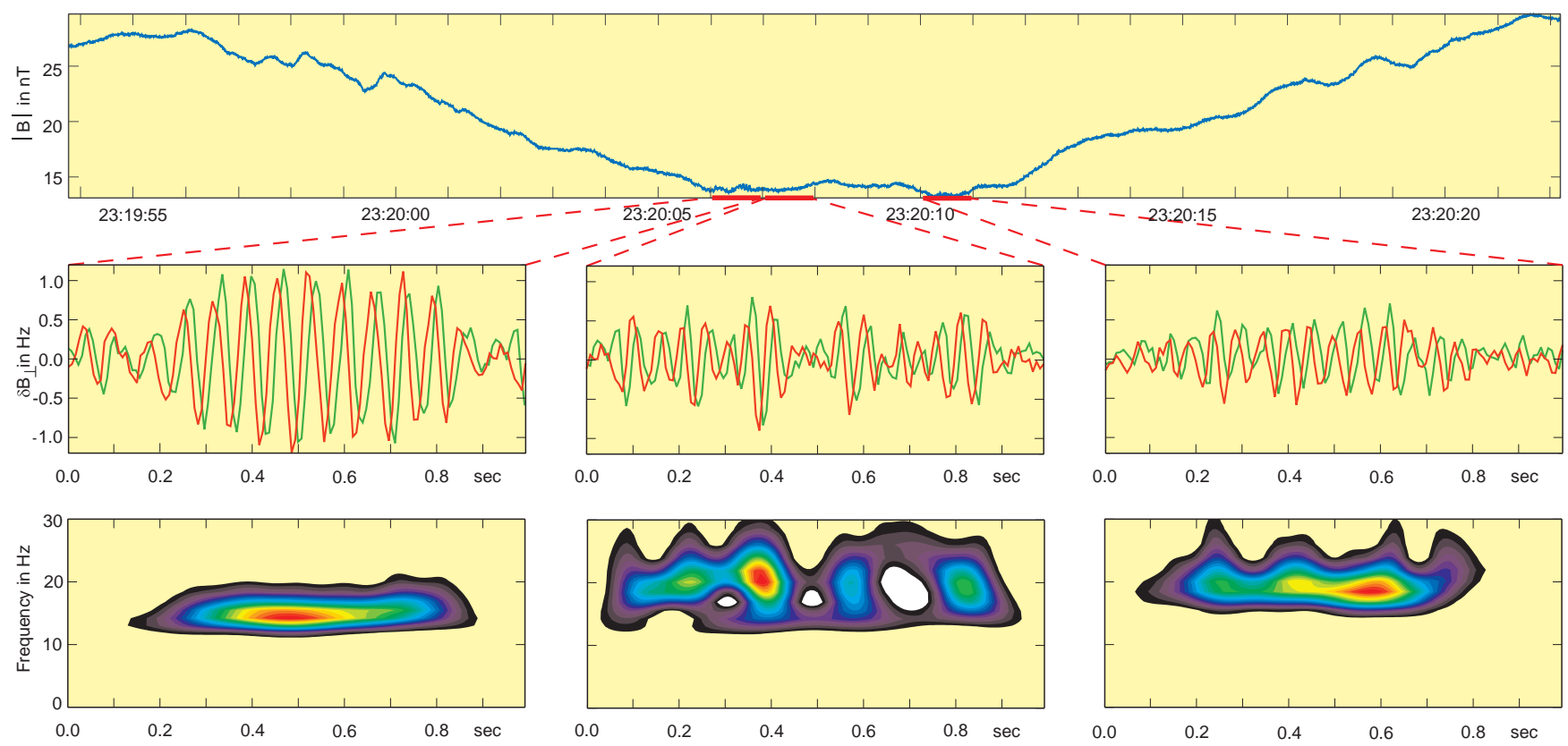

Fig. 1. Mirror mode trough, embedded lion roar wave forms perpendicular to the mean field, and right-hand polarized wavelet spectrograms 

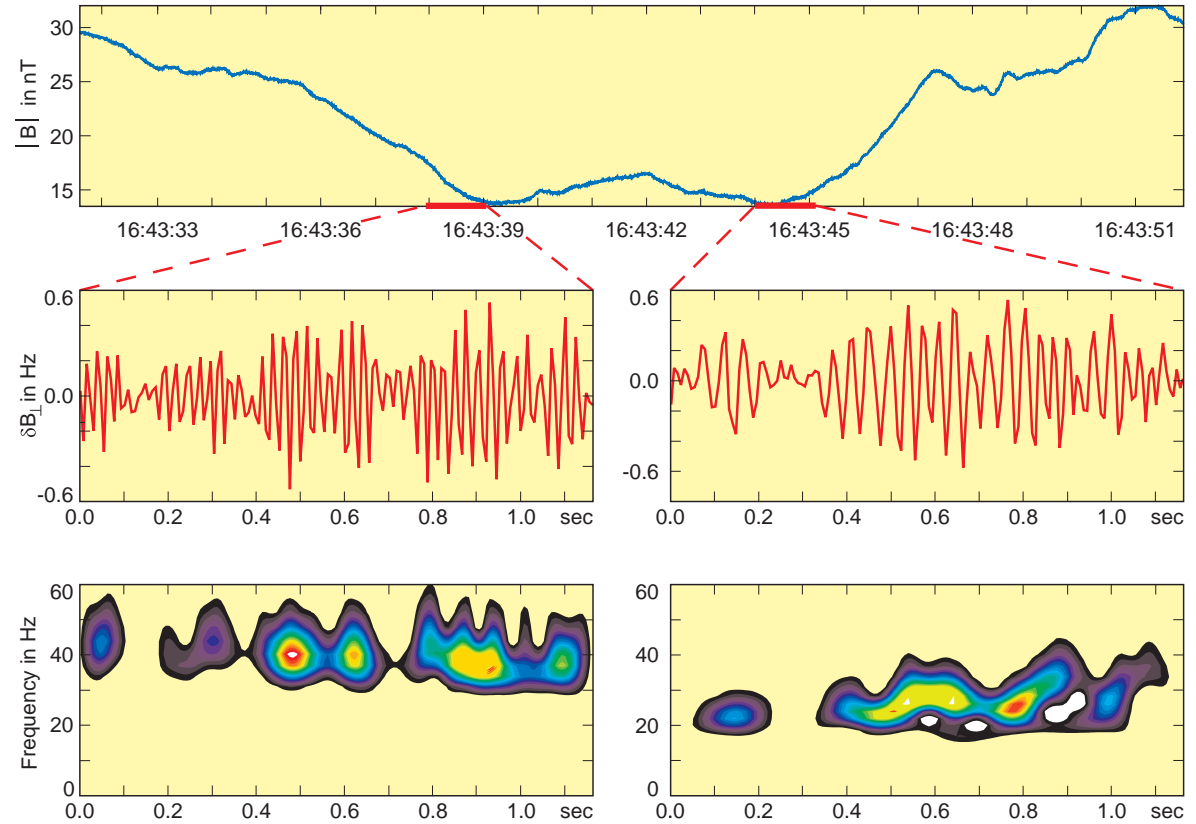

Fig. 2. Mirror mode trough, embedded lion roar wave forms, and right-hand polarized wavelet spectrograms trough detected on 28 January 1998. Both wave trains last about $1.2 \mathrm{~s}$ and contain four and two clearly discernible wave packets, respectively. They occur just $4 \mathrm{~s}$ apart on both sides of a stronger core field. While $f_{c e} \approx 420 \mathrm{~Hz}$ for both events, the lion roar frequency at 40 and $25 \mathrm{~Hz}$, respectively, is distinctly different for the two events. The cone angle between the wave vector and the ambient field varied between $0.1-0.6^{\circ}$ during the four wave packets of the first lion roar event, while it attained values of $0.25^{\circ}$ and $0.15^{\circ}$ during the first and second wave packet of the second event, respectively.

Another lion roar signal is shown in Fig. 3. It occurred on 5 March 1998 and this time the mirror trough did not contain a stronger core field. Again, the wave train lasts for about one second, but it shows considerable internal structure, with some wave packets lasting only for 3-4 wave cycles. In addition, one finds that the frequency varies during the event, from about $40 \mathrm{~Hz}$ to just above $20 \mathrm{~Hz}$, i.e., by a factor of two. In contrast, the electron gyrofrequency drops only slightly, from $360 \mathrm{~Hz}$ to $310 \mathrm{~Hz}$, during the event. The change in frequency proceeds in a series of glitches from packet to packet, both in upper and lower frequency cut-off. The cone angle, $\theta_{k b}$, ranged between $0.1^{\circ}$ and $0.4^{\circ}$ during the event.

\section{Statistics}

Including the six cases discussed above, we have analyzed the average properties of all 356 lion roar wave packets, which found in 4-months-worth of noonto-morning magnetosheath data in the frequency range between 8 and $64 \mathrm{~Hz}$. In particular we will look at the frequency and its dependence on the mirror trough background field, the duration of the lion roar wave packets, and the cone angle of the wave vector with the ambient field.
Figure 4 shows the occurrence distribution of the lion roar frequencies, normalized to the electron gyrofrequency, $f_{c e}$. The normalized frequencies are fairly evenly distributed in the range $0.05 \leq f / f_{c e} \leq 0.15$, with an average normalized frequency close to 0.1 . Case distribution as well as average value are not too different to those found by Zhang et al. (1998), except that we naturally lack the comparatively few samples in their higher-frequency tail due to our lower high-frequency limit.

The packet structure of the lion roars is clearly visible in most of the cases presented in the previous section, is
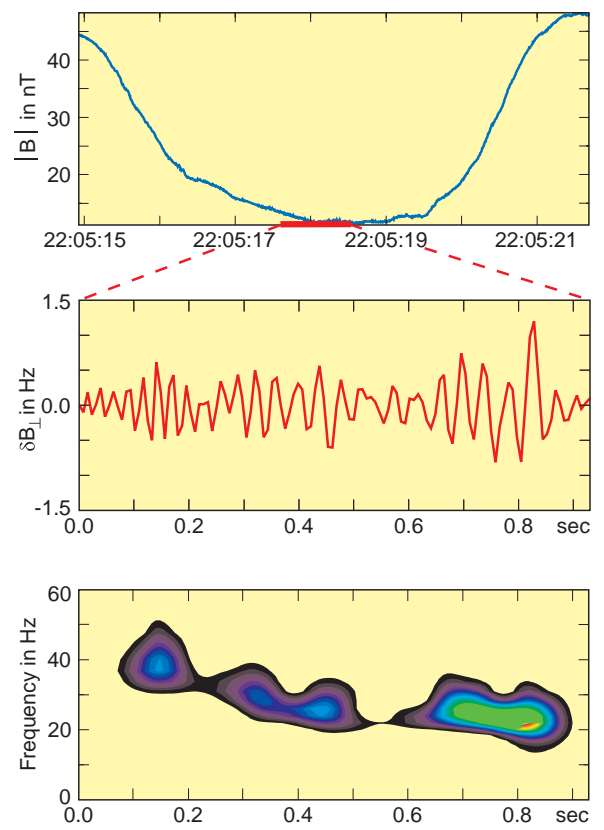

Fig. 3. Mirror mode trough, embedded lion roar waveform, and right-hand polarized wavelet spectrogram 


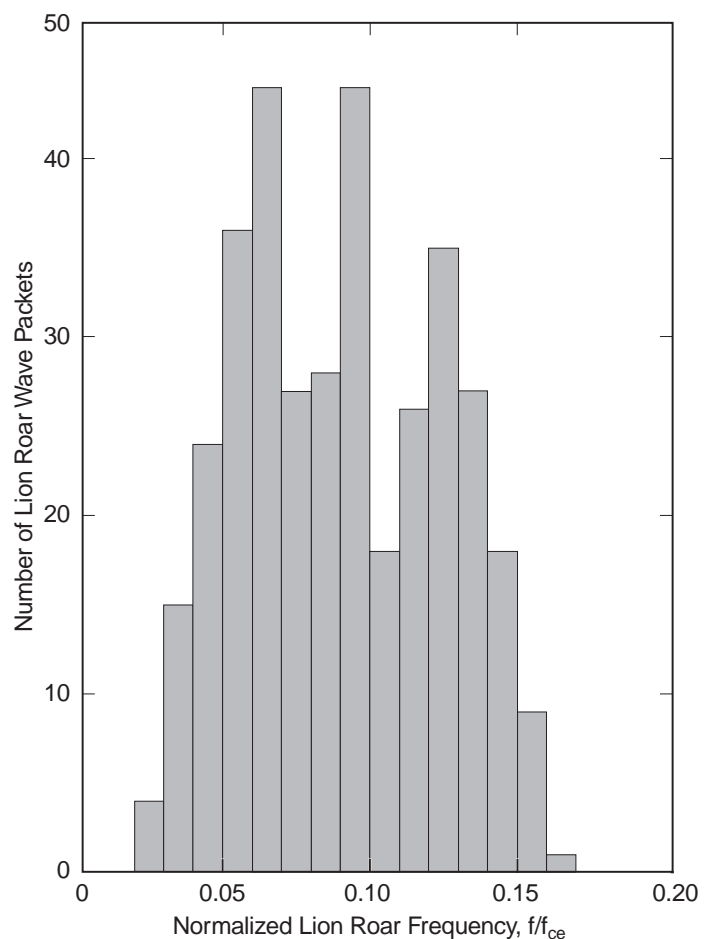

Fig. 4. Occurrence distribution of normalized lion roar frequencies

also found in the bulk of the 356 cases. Figure 5 gives the occurrence rates of the duration of the lion roar wave packets in number of wave cycles. Half of all cases have a wave packet length of less than five wave cycles and about $85 \%$ last less than ten wave cycles.

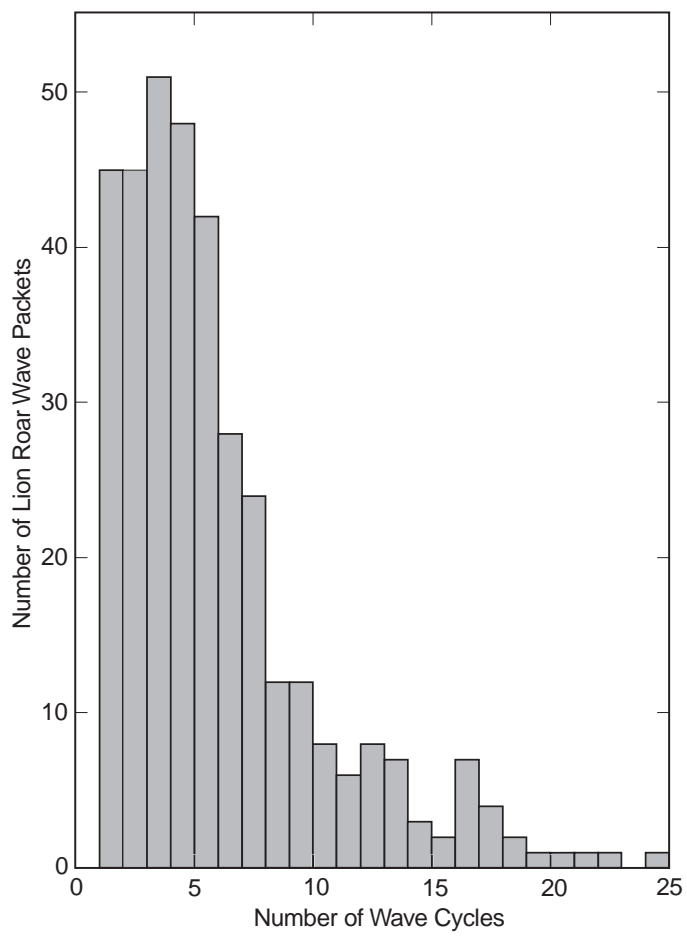

Fig. 5. Occurrence distribution of lion roar wave packet duration
As for the case studies, we have also performed a minimum variance analysis for all 356 cases and compared the wave vector direction with the ambient field direction. The occurrence distribution of the cone angle, $\theta_{k b}$, is given in Fig. 6. Except for ten cases with $1.5^{\circ} \leq \theta_{k B} \leq 5^{\circ}$, all cone angles are smaller than $1.4^{\circ}$. In fact, about half of the lion roar wave packets travel along the ambient magnetic field with cone angles smaller than $0.2^{\circ}$.

These values are definitely much smaller than the typical cone angles of $10-30^{\circ}$ found by Zhang et al. (1998) and Smith and Tsurutani (1976). Why? Looking into Fig. 7 of Fornacon et al. (1999), one notices that the ambient field direction easily changes by $5-20^{\circ}$ during a lion roar event lasting several seconds. We have done the minimum variance analysis on the wave packets with their average duration of $250 \mathrm{~ms}$ and compared the wave propagation direction with the average of the ambient field over the same period. We suspect that the authors of the earlier papers have used longer stretches of data for their minimum variance analysis and/or for averaging the background field direction.

\section{Summary}

The present study complements the earlier observations of lion roars, by extending the frequency range to lower values. While search coil magnetometers and plasma wave instruments are somewhat insensitive to the frequency range of some tens of Hertz, the Equator-S

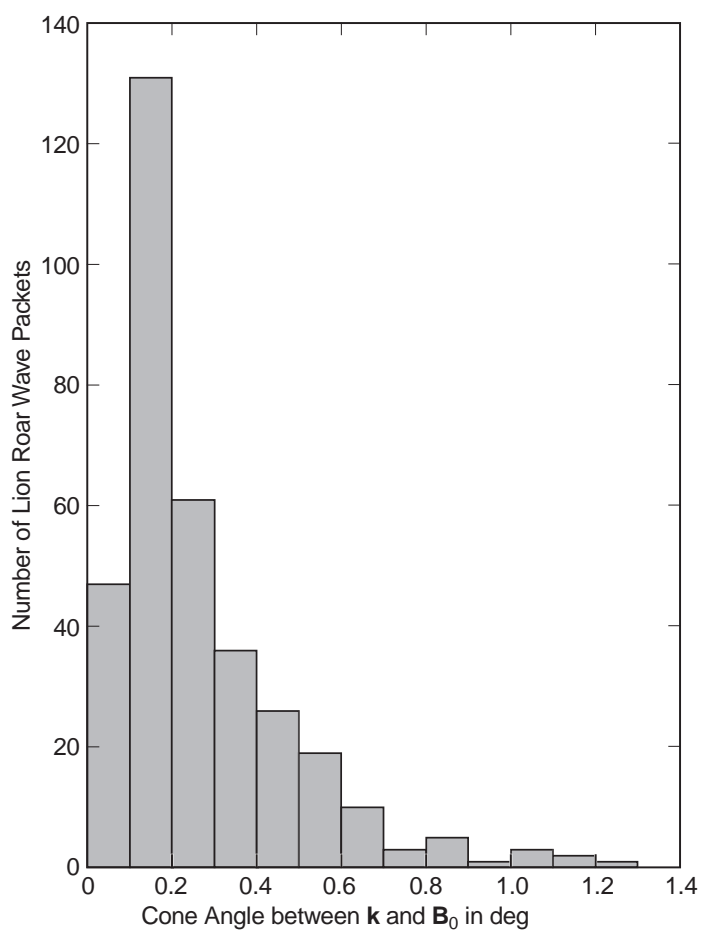

Fig. 6. Occurrence distribution between lion roar wave vector and ambient magnetic field. Ten more cases (between $1.5^{\circ}$ and $5^{\circ}$ ) have been omitted from this diagram 


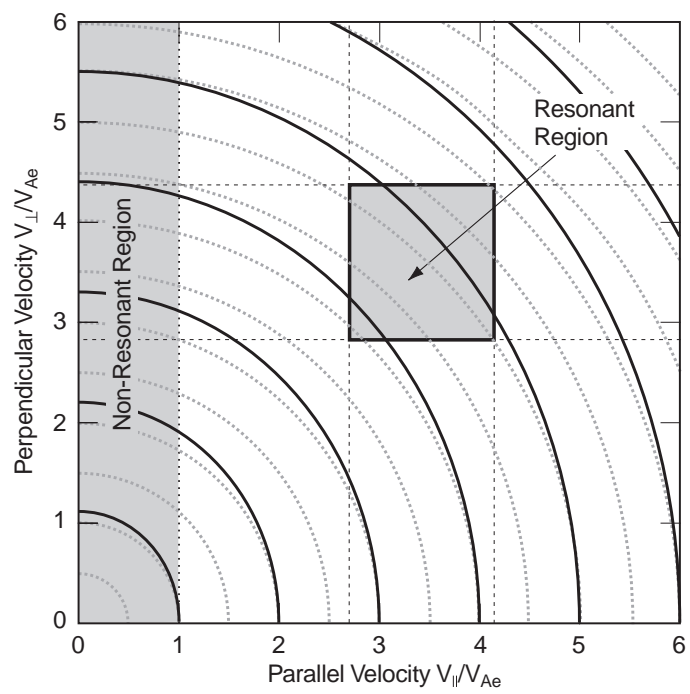

Fig. 7. The resonant electron velocity space as inferred from observations. The velocities are in units of the electron Alfvén velocity, $v_{A e}$. The vertical dashed line at $v_{\|}=1$ is the low energy boundary of the whistler resonance. The grey box shows the resonant region of electrons responsible for the emission of whistler waves. The solid ellipses are the iso-density contours of the weakly anisotropic electron distribution. The light circles show an isotropic distribution for comparison. Resonance takes place only in the narrow resonance region. As discussed in the text, nonlinear interaction may lead to the evolution of some residual bulge (not shown) in the resonant region which would be responsible for the observed emission

magnetometer, with a Nyquist frequency of $64 \mathrm{~Hz}$, cannot cover the higher frequency range, but our observations show that lion roar electron whistlers can be observed down to $10 \mathrm{~Hz}$, with similar characteristics as at higher frequencies, but also some new features:

1. Lion roars are near-monochromatic right-hand circularly polarized waves.

2. Lion roars have typical frequencies of $0.05-0.15 f_{c e}$.

3. Lion roars have typical amplitudes of $0.2-1.0 \mathrm{nT}$.

4. Lion roars have a wavelet-like packet structure with typical durations of 3-8 wave cycles;

5. Lion roars travel nearly parallel along the ambient magnetic field, more field-aligned than previously thought.

Average numbers for frequency, packet duration, and cone angle $\theta_{k B}$ are given in Table 1 .

\section{Discussion}

The most obvious observation is the narrow-bandedness of the signal. Since we know that it is in the right-hand

Table 1. Average frequency, wave packet duration, and cone angle between wave vector and ambient field for 356 cases

\begin{tabular}{lll}
\hline Quantity & Average & \\
\hline Center frequency & $f=33 \mathrm{~Hz}$ & $f / f_{c e}=0.09$ \\
Packet duration & $\Delta t=0.25 \mathrm{~s}$ & $f \Delta t=5.9$ cycles \\
Wave cone angle & $\theta_{k B}=0.33^{\circ}$ & \\
\hline
\end{tabular}

parallel whistler mode and is generated inside the magnetic trap configuration of a single mirror mode, parallel whistler instability theory applies. Under the assumption that the trapped electrons are anisotropic, dilute, and sufficiently energetic for justifying the application of linear theory, the upper frequency limit of the emission is expected at

$\frac{f_{\max }}{f_{c e}}=\frac{A_{e}}{A_{e}+1}$

The observed values for the frequency ratio suggests that the electron temperature anisotropy is of the order of $A_{e}=T_{\perp} / T_{\|}-1 \approx 0.1$ for all the events. On the other hand, we can take advantage of the whistler resonance condition

$\frac{v_{\| \text {res }}}{f_{c e} \lambda_{\|}}=\frac{f}{f_{c e}}-1$

which holds at both the upper and lower frequency limits. Writing it explicitly for these two limits we have

$v_{\| \min } / \lambda_{\| \min }=f_{\max }-f_{c e}, \quad v_{\| \max } / \lambda_{\| \max }=f_{\min }-f_{c e}$

In addition, the whistler dispersion relation imposes a condition on the parallel wave length

$\lambda_{\|}^{2}=\left(\frac{c}{f_{p e}}\right)^{2} \frac{f_{c e}}{f}$

Taken at the upper frequency cut-off, the resonance condition sets a lower limit on the parallel velocity of the resonant electrons

$\frac{v_{\| \min }}{v_{A e}}=\left(1-\frac{f_{\| \max }}{f_{c e}}\right)\left(\frac{f_{c e}}{f_{\| \max }}\right)^{1 / 2}$

which we estimate as $v_{\| \min } \approx 2.7 v_{A e}$, roughly three times the local electron Alfven speed (note that the waves and particles are antiparallel for resonance). In terms of the anisotropy the former expression reads

$\left|\frac{v_{\| \min }}{v_{A e}}\right|=\frac{A_{e}^{-1 / 2}}{A_{e}+1}$

Similarly, the lower frequency cut-off sets an upper limit on the resonant speed. Defining $\alpha=f_{\min } / f_{\max }$ as the ratio of the measured frequency cut-offs, one obtains

$v_{\| \max } / v_{\| \min }=\alpha^{-1 / 2}\left[A_{e}(1-\alpha)+1\right]^{3 / 2}$

Inserting the observed values yields

$v_{\| \max } \approx 1.53 v_{\| \min } \approx 4.14 v_{A e}$

The estimated anisotropy suggests that the perpendicular energy $\mathscr{E}_{\perp}$ of the resonant particles is comparable to their parallel energy, $\mathscr{E}_{\perp} \approx 1.1 \mathscr{E}_{\|}$. The magnetic energy per electron is given by $\mathscr{E}_{B}=m_{e} v_{A e}^{2} / 2$ yielding for the minimum parallel resonant energy $\mathscr{E}_{\| \min } \approx 7.3 \mathscr{E}_{B}$, and for the perpendicular energy of the resonant particles at instability threshold, $\mathscr{E}_{\perp \text { min }} \approx 8.0 \mathscr{E}_{B}$. Hence, the total energy of resonant electrons at threshold is $\mathscr{E}_{\min } \approx 15.3 \mathscr{E}_{B}$. Proceeding along the same lines for the resonant electrons at maximum resonant energy, we 
obtain the corresponding values $\mathscr{E}_{\| \max } \approx 17.1 \mathscr{E}_{B}$ for the maximum parallel resonant energy, $\mathscr{E}_{\perp \max } \approx 18.8 \mathscr{E}_{B}$ for the perpendicular electron energy at maximum resonance, and $\mathscr{E}_{\max } \approx 36.0 \mathscr{E}_{B}$ for the maximum total energy of the electrons at resonance. These estimates show that the resonant electrons cover the narrow energy range of

$15.3 \leq \frac{\mathscr{E}}{\mathscr{E}_{B}} \leq 36$

with $\mathscr{E}_{\max } / \mathscr{E}_{\min } \approx 2.35$ only.

Figure 7 shows the region of resonance in the $v_{\perp}-v_{\|}$ plane. The resonant region is a narrow rectangular domain instead of the vertically extended resonant strip expected in usual whistler resonance interaction. The vertical dashed line at $v_{\|}=v_{A e}$ is the low energy threshold boundary above that whistler instability may set on for positive anisotropy. Clearly the resonant region is sufficiently far above this boundary. Resonance seems to be possible only in a very narrow domain in velocity space and only a limited group of nearly monochromatic energy electrons contributes to wave growth. One possible reason is that these are just the electrons which can be trapped in the mirror wave field. The solid lines in the figure show the slight deviation of the electron iso-density contours from isotropy for a weak anisotropy of $A_{e} \approx 0.1$.

In order to check if the assumption of trapping is satisfied we can use the constancy of the magnetic moment $\mu=\mathscr{E}_{\perp} / B$ both at the field maximum and minimum. This leads to

$\frac{B_{\max }}{B_{\min }}=\frac{\mathscr{E}}{\mathscr{E} \perp} \approx \frac{15.3}{8} \approx 2$

which is roughly satisfied in the observations of the mirror modes for electrons mirroring close to the field maximum but still inside the mirror wave bottle.

The ratio of particle energy to magnetic energy per particle gives an idea about the value of the electron $\beta_{e} \approx \mathscr{E} / \mathscr{E}_{B}$, which is $\beta_{e} \approx 10$ in our case. Together with the estimated anisotropy $A_{e} \approx 0.1$ and referring to the numerical solutions of the whistler dispersion relation (cf., Gary, 1993) one finds from Fig. 8 that the maximum growth rate $\gamma$ of the whistler instability in our case is

$\gamma_{\max } \approx 10 \omega_{c i} \approx 14 \mathrm{rad} / \mathrm{s}$

From the definition of the whistler growth rate (see Hasegawa, 1975; Treumann and Baumjohann, 1997) we obtain

$\frac{\gamma}{2 \pi f_{c e}} \approx(2 \pi)^{1 / 2} \frac{n_{\mathrm{res}}}{n_{0}} \frac{v_{A e}}{v_{\|}}\left(\frac{f}{f_{c e}}\right)^{1 / 2}\left(1-\frac{f}{f_{c e}}\right)^{5 / 2}$

Inserting the measured values for the frequency ratio, $f / f_{c e} \approx 0.1$, taking $\quad v_{\|} \approx 3.5 v_{A e}$, and using $\gamma \approx 10 m_{e} / m_{i}=5.4 \times 10^{-3}$ for protons, we obtain for the fraction of resonant electrons that contributes to wave growth

$n_{\text {res }} / n_{0} \approx 0.03$

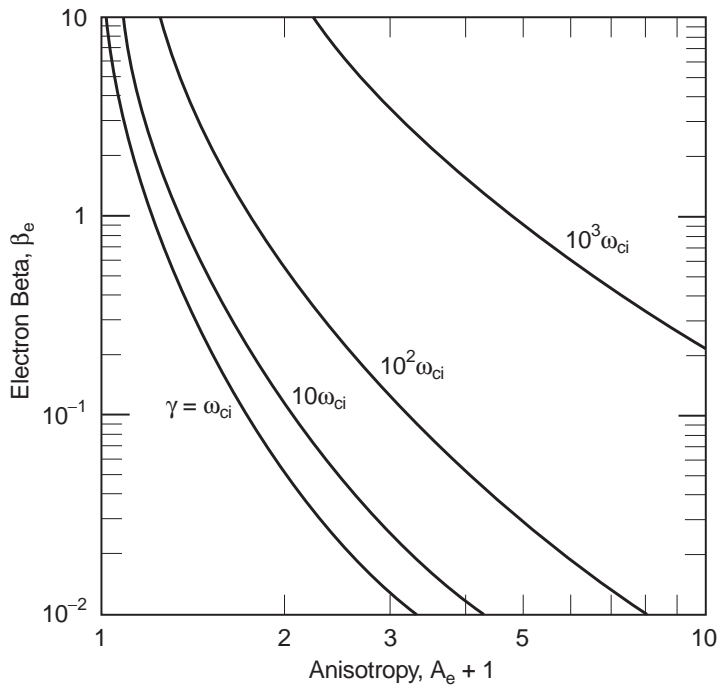

Fig. 8. Whistler growth rates in an anisotropic electron plasma as function of the temperature anisotropy $A_{e}+1$ and electron beta $\beta_{e}$ (after Gary, 1993). For the measured weak anisotropy $A_{e}=0.1$ and $1<\beta_{e}<10$ the growth rate is of the order of the ion cyclotron frequency $\gamma \approx \omega_{c i}$. The curves are drawn for $f_{p e} / f_{c e}=100$, close to that expected in the magnetosheath

implying that about $3 \%$ of the total electron population trapped in the mirror mode bottle develops the slight electron anisotropy which excites low frequency lion roars within the mirror modes. This observation raises the question why it is particularly the small group of electrons in the resonant region that drives the lion roar whistlers unstable. This question is not easily answered without measurement of the actual electron distribution function. Since such measurements are not available, we refer to the overall anisotropy of the electron distribution in this paper, as shown in Fig. 7. We show below that the whistlers have relatively large wave intensities. Under such conditions electrons in the resonant region will undergo diffusion from high $v_{\perp}$ towards higher $v_{\|}$. This may lead to the formation of a weak residual bulge on the electron distribution located in the resonant strip, similar to the ion-bulge distribution proposed for the nonlinear state of the mirror mode by Kivelson and Southwood (1996). The main electron distribution is then essentially isotropic.

The packet form of the emissions suggests that the whistler mode lion roar emissions reach the nonlinear state. In fact, the measured amplitude of $\delta B \approx 1 \mathrm{nT}$ corresponds to a magnetic wave energy density of

$\mathscr{E}_{w B}=|\delta B|^{2} / 2 \mu_{0} \approx 4 \times 10^{-13} \mathrm{Jm}^{-3}$

and a whistler wave electric field of

$\delta E_{w E} \approx v_{A e} \delta B_{w} \approx 1 \mathrm{mVm}^{-1}$

or a corresponding electric wave energy density of

$\mathscr{E}_{w} \approx \epsilon_{0}|\delta E|^{2} / 2 \approx 5 \times 10^{-14} \mathrm{Jm}^{-3}$

Compared to an estimated plasma thermal energy density of $n k_{B} T \approx 10^{-10} \mathrm{Jm}^{-3}$, these values correspond 
to fractions of $4 \times 10^{-3}$ and $5 \times 10^{-4}$, respectively. Waves of such an intensity will not only lead to the above mentioned quasilinear diffusion of electrons with excess perpendicular velocity towards parallel velocities. They will necessarily lead to nonlinear effects like modulational instability. Whistler wave amplitudes of $1 \mathrm{mV} / \mathrm{m}$ are rather high, of the same order as the convection electric field in the magnetosheath. It is therefore not unreasonable to assume that the original whistlers excited will nonlinearly evolve into packets being trapped in the modulations of the plasma. Since it is known that whistlers are trapped in overdense regions, the lion roar packets may be the wave signature of plasma compressions inside the mirror mode. Unfortunately, the lack of plasma measurements does not allow to check this suggestion.

The observation of glitches (Fig. 2) and gradual variations (Fig. 3) in the emission bands poses an interesting but difficult to resolve question. The easiest interpretation is the assumption of a time variation in the electron anisotropy, $A_{e}$. Such a variation may be caused by a decrease of the length of the mirror wave bottle during the time it takes the satellite to cross the bottle, which causes an increase in the parallel energy of the mirroring electron component, $\mathscr{E} \|$, and thus a decrease in $A_{e}$ and a shift to lower frequencies. The effective decrease in $A_{e}$ is very small, from $f_{\max } / f_{c e}=0.1$ to $f_{\max } / f_{c e}=0.05$ a decrease of only $\Delta A_{e}=0.01$ is required.

A similar decrease may also cause the apparent glitch in frequency in Fig. 2. If one assumes that essentially the same azimuthally drifting electron component is responsible for the emission, a shrinkage of the bottle in length during the passage of the satellite from left to right, causing a decrease in anisotropy of the above order, would explain the frequency glitch. This interpretation is, however, not unique. One cannot exclude the possibility that the two wave bands result from excitation in different remote parts of the bottle or even in different bottles. Since the waves are ideally parallel propagating waves, they could as well have migrated from a neighboring bottle into the one where they are observed.

Acknowledgements. Equator-S was financially supported via grant 50 OC 94024 by the German Space Agency, DARA (now DLR). The wavelet software was provided by C. Torrence and G. Compo, and is available at http://paos.colorado.edu/research/wavelets/.

The Editor-in-Chief thanks two referees for their help in evaluating this paper.

\section{References}

Fornacon, K.-H., H. U. Auster, E. Georgescu, W. Baumjohann, K.H. Glaßmeier, J. Rustenbach, and M. Dunlop, The magnetic field experiment onboard Equator-S and its scientific possibilities, Ann. Geophys., 17, 1999.

Gary, S. P., Theory of Space Plasma Microinstabilities, p. 138, Cambridge Univ. Press, Cambridge, 1993.

Hasegawa, A., Plasma Instabilities and Nonlinear Effects, p. 81, Springer, Berlin Heidelberg New York, 1975.

Kivelson, M. G., and D. J. Southwood, Mirror instability II: the mechanism of nonlinear saturation, J. Geophys. Res., 101, 17,365-17,371, 1996.

Lui, A. T. Y., and A. -H. Najmi, Time-frequency decomposition of signals in a current disruption event, Geophys. Res. Lett., 24, 3157-3160, 1997.

Smith, E. J., and B. T. Tsurutani, Magnetosheath lion roars, J. Geophys. Res., 81, 2261-2266, 1976.

Torrence, C., and G. P. Compo, A practical guide to wavelet analysis, Bull. Am. Met. Soc., 79, 61-78, 1998.

Treumann, R. A., and W. Baumjohann, Advanced Space Plasma Physics, p. 109, Imperial College Press, London 1997.

Tsurutani, B. T., E. J. Smith, R. R. Anderson, K. W. Ogilvie, J. D. Scudder, D. N. Baker, and S. J. Bame, Lion roars and nonoscillatory drift mirror waves in the magnetosheath, J. Geophys. Res., 87, 6060-6072, 1982.

Zhang, Y., H. Matsumoto, and H. Kojima, Lion roars in the magnetosheath: the Geotail observations, J. Geophys. Res., 103, 4615-4626, 1998. 